\title{
The Use of Vibrating Wire Technique for Precise Positioning of CESR Phase III Super- Conducting Quadrupoles at Room Temperature. *
}

\author{
A. Temnykh ${ }^{\dagger}$ \\ Laboratory of Nuclear Studies, Cornell University, Ithaca NY 14953, USA
}

\section{Abstract}

Vibrating wire technique has been used for precise positioning of CESR Phase III quadrupole magnets in cryostats at room temperature. The alignment was done at $8 \times$ $10^{-2} \mathrm{~T} / \mathrm{m}$ of gradient of magnetic field at quadrupole magnets. The position of the magnetic center of the quadrupoles was measured with $0.03 \mathrm{~mm}$ precision. The magnets were aligned with cryostat centerline with better than $0.2 \mathrm{~mm}$ precision.

The measurement setup, instruments and the procedure are described.

\section{INTRODUCTION}

CESR Phase III upgrade calls for installation of four final focusing super-conducting (SC) quadrupole magnets around interaction point (IP), see [3]. Two SC quadrupoles sharing common cryostat will be placed on each side of IP. The position of SC magnets will not be available for survey after they are placed inside of cryostats and cooled down. Thus the magnets should be precisely aligned with cryostats center at the moment of installation. Afterward, well defined references on cryostat's body can be used for precise positioning relative to global survey system.

The most direct way to align the magnet with cryostat is to measure it's magnetic center position and, using adjustment in the suspension mechanism, to align magnetic centers with cryostat center line. Because the adjustment mechanism is accessible only at room temperature, the magnet positioning should be done in warm state and then the magnetic center position should be verified again at low temperature. At room temperature the quadrupole coils have significant resistivity and the current through the magnet and the magnetic field gradient are very limited by magnet heating. It means that, the technique used for finding the quadrupole magnetic center must have high spatial resolution at very low magnetic field gradient. Suitable method, called "Vibrating wire technique" was developed in [1]. It is based on the following phenomenon. The Lorenz forces between alternating current flowing through the taut wire and transverse magnetic field excite a mechanical wire vibration. The vibration is especially strong if the driving current frequency is in resonance with one of the modes of wire vibration. The amplitude and the phase of vibration relative to the driving current depend on the magnetic field distribution along the wire. Measuring the phase and amplitude at various frequencies one can reconstruct

\footnotetext{
* Work supported by National Since Foundation

$\dagger$ e-mail: abt6@cornell.edu
}

the field distribution.

In [2] using a prototype magnet, it was demonstrated that the technique can be used for the required type of alignment.

\section{SETUP DESCRIPTION}

The arrangement of the magnets and measurement setup is shown in Figure 1.

Two super-conductive quadrupoles, $Q 1$ and $Q 2$, of $65 \mathrm{~cm}$ length were located inside a cylindrical cryostat. Because of significant resistivity of the magnet coils at room temperature, the current through the magnets during measurements was limited to $2 \mathrm{~A}$. At this current magnetic field gradient was $7.92 \times 10^{-2} \mathrm{~T} / \mathrm{m}$.

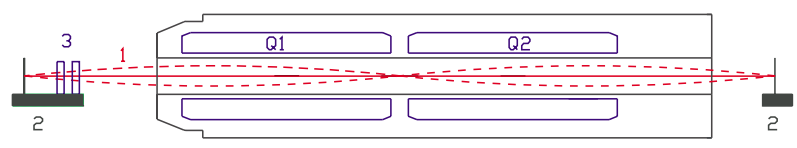

Figure 1: The measurement setup. 1 - probe (vibrating) wire, dashed line shows the mode of vibration used in measurement. Q1, Q2 - super-conductive quadrupoles inside of cryostat. 2 - stages movable in horizontal and vertical planes. 3 - horizontal and vertical wire position sensors.

The $100 \mu \mathrm{m}$ diameter, $3.02 \mathrm{~m}$ length copper-beryllium gold coated wire (1), referred hereafter as either the probe or the vibrating wire, was stretched through the cryostat bore. The wire ends have been fixed on the stages (2) movable in horizontal and vertical planes with micro-screws driven by stepping motors. Two photo-transistor-LED assemblies (3) placed on the left stage detected vertical and horizontal wire vibration. A digital wave form generator HP33120A was used to drive AC current through the wire. For a signal analysis as well as for control of digital wave form generator and stepping motors, a Macintosh Quadra 800 computer with application programs created in LabVIEW has been used.

Similar to the experiments described in [2], in order to reduce the effect of background magnetic field, the second wire vibration mode shown by dashed line on Figure 1 was chosen for the measurement. The wire middle point, the node of the mode, was right in the middle between $Q 1$ and $Q 2$. This provided equal sensitivity of the measurement for $Q 1$ and $Q 2$ misalignment. 


\section{PROBE WIRE POSITION DETERMINATION}

To establish the probe wire position in relation to the cryostat central line four dowel pins placed at precisely defined positions on both ends of the cryostat have been used. The centers of the pins were located exactly in the vertical and horizontal mid-plane of the cryostat. Electrical contact between probe wire and thin wires stretched between the pins has been used to determine probe wire position. The error in the probe wire position established in this way was smaller then $0.025 \mathrm{~mm}$. Note that in the horizontal plane the probe wire can be aligned with cryostat centerline over all cryostat length, in vertical plane, because of sag, it can be centered only at two points. In our case these were cryostat ends.

\section{VERTICAL SAG EFFECT CONSIDERATION}

Vertical sag of the wire aligned with cryostat centerline at cryostat ends can be expressed as:

$$
y_{s a g}(z)=-\frac{g}{8\left(l_{w} f_{1}\right)^{2}}\left(z-z_{f}\right)\left(z-z_{r}\right)
$$

Here, $y_{\text {sag }}$ is the vertical wire displacement relative to cryostat center line, $z$ is the distance from wire end, $g$ is gravity, $l_{w}$ - wire length, $f_{1}$ - is the fundamental mode frequency of the wire vibration, $z_{f}$ and $z_{r}$ are longitudinal coordinates of front and rear cryostat ends.

The measured parameter, $a_{2 x, y}$, defined in the reference [1] is proportional to the second harmonic of the Fourier sine transform of magnetic field along wire. Index " 2 " indicates the second wire vibration mode, $x, y$ are referring to the wire vibration in horizontal and in vertical plane. Note that vibration in vertical plane is excited by horizontal magnetic field and vice versa. For a quadrupole magnet aligned with the cryostat center, with gradient $G$, length $l_{m}$, and located at $z=z_{m}$, parameter $a_{2 y}$ will be:

$$
a_{2 y} \propto=\int_{z_{m}-0.5 l_{m}}^{z_{m}+0.5 l_{m}} G y(z) \sin \left(\frac{2 \pi z}{l_{w}}\right) d z
$$

Here $y(z)$ is the vertical displacement of a probe wire relative to the cryostat center. If both ends of the probe wire are shifted up by $\delta y, y(z)$ will be sum of sag and the shift:

$$
y(z)=y_{\text {sag }}(z)+\delta y
$$

Substituting equation (3) in (2) and taking into account equation (1), one can find the distance $\delta y$ at which the probe wire ends should be shifted up to make $a_{2 y}$ equal zero:

$$
\delta y=\frac{g}{8\left(l_{w} f_{1}\right)^{2}} \frac{\int_{z_{m}-0.5 l_{m}}^{z_{m}+0.5 l_{m}}\left(z-z_{f}\right)\left(z-z_{r}\right) \sin \left(\frac{2 \pi z}{l_{w}}\right) d z}{\int_{z_{m}-0.5 l_{m}}^{z_{m}+0.5 l_{m}} \sin \left(\frac{2 \pi z}{l_{w}}\right) d z}
$$

\begin{tabular}{|lll|}
\hline \hline Probe wire length & $l_{w}$ & $302.2 \mathrm{~cm}$ \\
Front reference wire position & $z_{f}$ & $53.3 \mathrm{~cm}$ \\
Rear reference wire position & $z_{r}$ & $276.8 \mathrm{~cm}$ \\
Fundamental mode frequency & $f_{1}$ & $32.7 \mathrm{~Hz}$ \\
$Q 1$ and $Q 2$ length & $l_{m}$ & $65 \mathrm{~cm}$ \\
$Q 1$ center position & $z_{m}$ & $105.5 \mathrm{~cm}$ \\
$Q 2$ center position & $z_{m}$ & $196.7 \mathrm{~cm}$ \\
$Q 1$ vertical sag correction & $\delta y$ & $0.100 \mathrm{~mm}$ \\
$Q 2$ vertical sag correction & $\delta y$ & $0.136 \mathrm{~mm}$ \\
\hline
\end{tabular}

Table 1: Vertical sag correction.

This is the correction we have to apply to compensate the vertical sag effect.

For a short magnet, $l_{m}<<l_{w} / 2$, equation (4) gives:

$$
\delta y=\frac{g}{8\left(l_{w} f_{1}\right)^{2}}\left(z_{m}-z_{f}\right)\left(z_{m}-z_{r}\right)
$$

Comparing this formula with equation (1) one can see that in this case the correction, $\delta y$, is the sag at the center of the magnet.

Table 1 shows parameters of the setup and calculated sag correction. To compensate the sag when aligning the $Q 1$ magnet, the wire ends should be moved up by $0.100 \mathrm{~mm}$. For the $Q 2$ alignment the ends should be moved up by $0.136 \mathrm{~mm}$.

\section{MEASUREMENT AND MAGNET POSITIONING}

The alignment procedure for both cryostats, cryostat " 1 " and cryostat " 2 ", was identical and consisted of the following steps.

The first step was to establish the probe wire position as described above. Then by scanning the probe wire in vertical and horizontal planes and measuring parameters $a_{2 x, y}$ with and without current in the magnets, the precise positions of the magnetic centers of the $Q 1$ and $Q 2$ before adjustment were determined. The measurement is illustrated on the two upper plots in Figure 2. On the plots are measured $a_{2 x, y}$, as a function of probe wire position in vertical and horizontal plane relative to cryostat centerline. Solid dots represent background measurement, i.e., with no current in $Q 1$ and $Q 2$. Triangles and squares are for $2 \mathrm{~A}$ current in $Q 1$ and in $Q 2$ respectively. The probe wire crosses magnetic center of quadrupoles in location where $a_{2 x, y}$ measured with the current are equal to background. Linear fit of the data corrected by sag effect gave the precise position of $Q 1$ and $Q 2$ magnetic center.

The next step was the magnet position adjustment. For that the probe wire was placed at cryostat center line in horizontal plane, in vertical plane it's ends were placed slightly higher to correct for the sag effect according to the above discussion. The frequency of the current through the probe wire was set close to the resonance of the second harmonic wire vibration. Comparing signals from the wire position detectors showing amplitude of the wire vibration, with and 

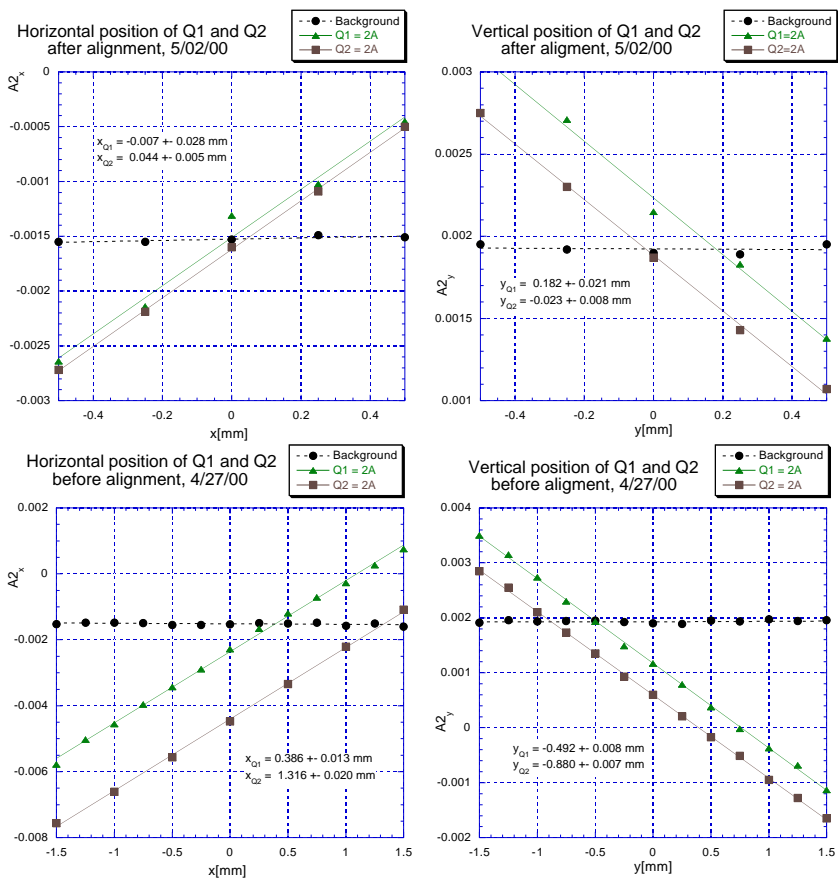

Figure 2: . Measurement of $Q 1$ and $Q 2$ ( cryostat 2) magnetic center position before and after alignment. Here shown parameters $a_{2 x, y}$ as a function of horizontal $(x)$ and vertical $(y)$ probe wire position relative cryostat center.

without current through the magnets, it was possible to estimate how far the magnetic centers of the quadrupoles was from the desired position. A few iterations of the measurements and adjustments of the magnet supporting system were needed to put the magnets in position such that the switching the current through the magnets off and on produced no effect on wire vibration. After that, the precise measurement of magnetic centers was repeated. Two lower plots of figure 2 and data in table 2 show the result of the measurement. Note that vertical magnet positions listed in the table 2 were obtained from the data plotted on figure 2 using sag correction presented in table 1 .

It should be mentioned that the precise measurement of the magnetic center position by the probe wire scan took approximately 15 minutes. The position estimation by comparing wire vibration amplitude for magnets turned on and off took just a few seconds.

Result of the magnetic center position measurement after tuning for all four magnets is presented in table 2 . Before tuning, the average magnet displacement relative to the cryostat center was about $1 \mathrm{~mm}$. After tuning, the measured displacement of three of four magnets is less than $0.1 \mathrm{~mm}$. Vertical alignment of $Q 2$ magnet in cryostat " 2 " was limited mechanically by its supporting system. This magnet has been positioned at $0.159 \mathrm{~mm}$ below the cryostat center. The possible errors of the measured location of magnetic center is in the range of 0.01 and $0.03 \mathrm{~mm}$.

\begin{tabular}{|c|c|c|}
\hline \hline Cryostat & \multicolumn{2}{|c|}{ Position after tuning } \\
Magnet & $x[\mathrm{~mm}]$ & $y[\mathrm{~mm}]$ \\
\hline "1" & & \\
Q1 & $0.013 \pm 0.022$ & $-0.001 \pm 0.014$ \\
Q2 & $0.065 \pm 0.032$ & $0.026 \pm 0.027$ \\
\hline "2" & & \\
Q1 & $-0.007 \pm 0.028$ & $0.082 \pm 0.021$ \\
Q2 & $0.044 \pm 0.005$ & $-0.159 \pm 0.008$ \\
\hline
\end{tabular}

Table 2: Position of magnetic center of $Q 1$ and $Q 2$ quadrupoles in cryostat " 1 ", and in cryostat " 2 " after alignment

\section{CONCLUSION}

The vibrating wire technique has been used for precise positioning of the CESR Phase III super-conducting quadrupole magnets inside of cryostats at room temperature. As a result, three of the four magnets have been aligned with the cryostat center with better than $0.1 \mathrm{~mm}$ precision, the fourth magnet has been positioned at $0.159 \mathrm{~mm}$ off the cryostat center because of limitation in the range of motion. The precision of the magnetic center position measurement was better then $0.03 \mathrm{~mm}$.

The vibrating wire technique has been proved to be a very convenient and adequate tool for the precise alignment of the quadrupole magnets with low gradient.

\section{ACKNOWLEDGMENT}

I would like to thank David Rice and David Rubin for their attention to this work, Scott Chapman for help in the checking of the position of the probe wire. I am also very grateful to James Welch for his cooperation and for his help with the precise movement of the magnets.

\section{REFERENCES}

[1] A. Temnykh, Vibrating wire field-measuring technique, Nuc. Inst., A 399 (1997) 185-194

[2] A. Temnykh, The Magnetic Center Finding using Vibrating Wire Technique, Preperint CBN 99-22.

[3] J. Welch, G.F Dugan, E. Nordberg, D. Rice, The Superconducting Interaction Region Magnet System for the CESR Phase III Upgrade. Proc. 1997 Particle Accelerator Conference, vol. 3, pp. 3383-3385 